\title{
WDR34 mutation from anencephaly patients impaired both SHH and PCP signaling pathways
}

\author{
Hailing Yin ${ }^{1,2} \cdot$ Rui Peng ${ }^{1} \cdot$ Zhongzhong Chen ${ }^{1} \cdot$ Hongyan Wang ${ }^{1,3} \cdot$ Ting Zhang ${ }^{4} \cdot$ Yufang Zheng $\mathbb{D}^{1,2,3}$
}

Received: 18 December 2019 / Revised: 11 June 2020 / Accepted: 11 June 2020 / Published online: 23 June 2020

(c) The Author(s), under exclusive licence to The Japan Society of Human Genetics 2020

\begin{abstract}
Neural tube defects (NTDs) are debilitating human congenital abnormalities due to failure of neural tube closure. Sonic Hedgehog (SHH) signaling is required for dorsal-ventral patterning of the neural tube. The loss of activation in SHH signaling normally causes holoprosencephaly while the loss of inhibition causes exencephaly due to failure in neural tube closure. WDR34 is a dynein intermedia chain component which is required for SHH activation. However, Wdr34 knockout mouse exhibit exencephaly. Here we screened mutations in WDR34 gene in 100 anencephaly patients of Chinese Han population. Compared to 1000 Genome Project data, two potentially disease causing missense mutations of WDR34 gene (c.1177G >A; p.G393S and c.1310A>G; p.Y437C) were identified in anencephaly patients. These two mutations did not affect the protein expression level of WDR34. Luciferase reporter and endogenous target gene expression level showed that both mutations are lose-of-function mutations in SHH signaling. Surprisingly, WDR34 could promote planar cell polarity (PCP) signaling and the G393S lost this promoting effect on PCP signaling. Morpholino knockdown of wdr34 in zebrafish caused severe convergent extension defects and pericardial abnormalities. The G393S mutant has less rescuing effects than both WT and Y437C WDR34 in zebrafish. Our results suggested that mutation in WDR34 could contribute to human NTDs by affecting both SHH and PCP signaling.
\end{abstract}

\section{Introduction}

Neural tube defects (NTDs) are structural birth defects of brain and spinal cord. The incidence of NTD ranges 15.3-23.0 per 10,000 live birth worldwide [1-3]. In the past decades, the prevalence rate of NTD is much higher in Shanxi province of China $[4,5]$. NTDs have resulted from a

Supplementary information The online version of this article (https:// doi.org/10.1038/s10038-020-0793-z) contains supplementary material, which is available to authorized users.

Yufang Zheng

zhengyf@fudan.edu.cn

1 Obstetrics \& Gynecology Hospital, Institute of Reproduction \& Development, Fudan University, Shanghai, China

2 Institute of Developmental Biology \& Molecular Medicine, Fudan University, Shanghai, China

3 State Key Laboratory of Genetic Engineering, Collaborative Innovation Center of Genetics and Development, School of Life Sciences, Fudan University, Shanghai, China

4 Capital Institute of Pediatrics, Beijing, China failure of neural tube closure (NTC). NTC occurs during early gestation stage (3rd and 4th weeks). Based on different regions of unclosed neural tube, NTDs have multiple clinical features including anencephaly, exencephaly, myelomeningocele, and spina bifida [6]. The infants of anencephaly or exencephaly often are stillborn or die shortly after birth, whereas spina bifida patients may survive but suffer severe and lifelong physical disabilities [3]. Both genetic and environmental factors contribute to the etiology of NTDs [7].

The primary cilium is a hair-like sensory organelle present on the surface of most vertebrate cell types and is required for many essential signaling processes such as Hedgehog $(\mathrm{HH})$ and WNT signaling $[8,9]$. The construction and function of cilia depends on microtubule-based motor proteins and intraflagellar transport (IFT) system inside cilia. IFT complexes move along the axonemal microtubules to transport cargo between the base and the tip of the cilium. Kinesin-2 is the anterograde motor to move IFT complexes from the base to the tip of cilia; while cytoplasmic dynein-2 is the retrograde motor to move IFT from the tip to the base of cilia $[9,10]$. Cytoplasmic dynein2 complex contains multiple proteins, including the 
cytoplasmic dynein-2 heavy chains, light chains, intermediate chains, and light intermediate chains [10, 11]. Many mutations in the subunits of those motor complexes have been identified in human ciliopathies $[12,13]$.

WD repeat domain 34 (WDR34), a member of the WD repeat protein family, is a dynein- 2 intermediate chain protein and are required for dynein-2 function [14, 15]. Defects in WDR34 gene have been associated with ciliopathies such as Short-rib thoracic dysplasia 11 (SRTD11) with or without polydactyly (OMIM number *613363) $[16,17]$.

Cilium is the key for the Sonic Hedgehog (SHH) signaling, which plays essential roles for patterning the dorso-ventral axis of the neural tube [18-20]. Many mice mutants of SHH signaling pathway components have been established, which is nicely summarized by Murdoch and Copp [21]. In brief, the loss of activation of SHH signaling causes holoprosencephaly but not NTDs, while the loss of inhibition of SHH signaling causes exencephaly, a subtype of NTDs [21]. For example, the SHH signaling negative regulator Sufu knockout mouse has open neural tube [22]; and a Gli3 mutant (Pdn/Pdn) has both polydactyly and exencephaly phenotype [23, 24]. Recently, a Wdr34 knockout mouse model was reported [25]. Indeed, lack of $W d r 34$ in mice caused defects in HH signaling and retrograde ciliary protein trafficking. The $W d r 34^{-1-}$ mutant mice exhibit polydactyly, which is a characteristic ciliopathy phenotype. Interestingly, the reported $W d r 34^{-1-}$ mutant mice also exhibit exencephaly [25]. This phenotype is contradictory to the function of Wdr34 in activation of SHH signaling. Therefore, the function of WDR34 during neural tube development deserves a further investigation.

In this study, we identified two potential disease causing mutations (c.1177G $>$ A; p.G393S and c.1310A $>$ G; p. Y437C) in WDR34 among a Chinese NTD cohort with mostly anencephaly cases [26]. G393S is not identified in the Chinese Han population from 1000 Genome Project [27]. This mutation indeed lost activation on SHH signaling. To our surprise, WDR34 could also activate PCP signaling and the G393S is a loss-of-function mutation in PCP signaling. Furthermore, G393S had less rescuing ability compared to WT and Y437C WDR34 in zebrafish. Our data suggested that WDR34 is involved in not only SHH but also PCP signaling, which is required for proper NTC.

\section{Materials and methods}

\section{Study subjects}

NTD samples from aborted fetuses with severe cranial phenotypes, e.g., anencephaly, were collected from 1992 to
2011. A total of 100 NTD samples ( $94 \%$ are anencephaly; $60 \%$ male, $37 \%$ female, $3 \%$ unknown) were collected from Shanxi province (Table S1). All samples were collected with the approval of the institutional ethics committee of Capital Institute of Pediatrics Beijing and Fudan University (\#Kyy2018-56 to YZ). The informed consents were signed by the parents of subjects.

\section{DNA Sequencing and data analysis}

WGS on NTD cases were performed as previously described [26]. To confirm the genotyping results of WDR34 from WGS, the region containing the missense mutations of WDR34 within NTD samples were amplified by PCR and sent for Sanger sequencing. WDR34 variants were checked in two public databases: the 1000 Genome Project [27], and the gnomAD [28]. Mutations were also evaluated by using PolyPhen-2 [29] (http://genetics.bwh.harvard.edu/pph2/) and SIFT [30, 31] (http://provean.jcvi.org/) programs.

\section{Plasmids and site-directed mutagenesis}

Human WDR34 was cloned in pCMS-EGFP vector and the plasmid was kindly provided by Prof. Zhiheng Xu of Chinese Academy of Science. Mutations to WDR34 plasmids were generated using the KOD-Plus-Mutagenesis Kit (TOYOBO, Japan). The primers used for site-directed mutagenesis are as follows: G393S-Forward: $5^{\prime}$-ttc tcc cec cac ggc agt cce atc tac tct g-3', G393S-Reverse: $5^{\prime}$-cag agt aga tgg gac tgc cgt ggg ggg aga a-3', Y437C-Forward: $5^{\prime}$-agc tct ccc tca agt gtc tgt ttg ctg tgc g-3', Y437C Reverse: $5^{\prime}$-cgc aca gca aac aga cac ttg agg gag agc $\mathrm{t}-3^{\prime}$. All plasmids were verified by DNA sequencing. TOPFlash-luciferase reporter plasmid was obtained from Upstate Biotechnology Inc., now Millipore, USA. pFR-luciferase reporter and pFA22-c-Jun plasmids were obtained from Agilent technology, Santa Clara, CA, USA. DNA concentrations were examined by using NanoDrop 2000 (Thermo Fisher Scientific, USA).

\section{In vitro transcription of amplified cDNA}

The WDR34 vectors were linearized with NotI restriction enzyme (New England Biolabs, Ipswich, MA, USA) and in vitro transcribed with the Sp6 mMESSAGE mMACHINE kit (Thermo Fisher Scientific, USA). The reaction was carried out at $37{ }^{\circ} \mathrm{C}$ for $2 \mathrm{~h}$, followed by the addition of DNase I and incubation for $15 \mathrm{~min}$. RNA was isolated by phenol/chloroform extraction and 2-propanol precipitation. After centrifugation, the RNA pellet was re-suspended in RNase-free water, and the quantity and purity were determined by UV spectrophotometry and electrophoresis. 


\section{Zebrafish embryo microinjections}

The wild-type (WT) AB zebrafish strain was maintained and bred under standard conditions. The WDR34 mRNA was diluted in RNA-free water. Morpholino oligos (MOs) for $w d r 34$ were same to the previous report [32]. To examine their effect of rescuing, we injected $2.3 \mathrm{nl}$ of the following solution into zebrafish embryos during the 1-2 cell stage. The injected solutions were $50 \mathrm{ng} / \mu \mathrm{l}$ or $100 \mathrm{ng} / \mu \mathrm{l}$ mRNA of WT or WDR34 mutants $(115 \mathrm{pg}$ or $230 \mathrm{pg}$ mRNA was injected for each mRNA) mixed with $0.3 \mathrm{mM}$ $w d r 34$ MOs, respectively. In the overexpression experiment, $2.3 \mathrm{nl}$ of each mRNA was injected at a concentration of $50 \mathrm{ng} / \mu \mathrm{l}$, resulting of $115 \mathrm{pg}$ mRNA injected into each zebrafish embryo during the 1-2 cell stage. After injection, the embryos were allowed to develop at $28.5^{\circ} \mathrm{C}$ for $48 \mathrm{~h}$. Phenotypes were observed by microscopy and scored. And images were taken using a Leica MZ95 microscope system. Statistical significance was calculated using online twotailed Fisher's exact test tool (www.langsrud.com/stat/ fisher.htm). Both zebrafish wdr34 MOs and control MOs were purchased from Gene Tools, LLC. The sequences of zebrafish wdr34 MOs and control MOs are listed below:

Control MO 5'-CCT CTT ACC TCA GTT ACA ATT TAT A-3'

$w d r 34$ MO $5^{\prime}$-ACA ATA AAC TGT TAC CGG CCA AAT C-3'

\section{Cell culture and luciferase assay}

HEK293T cells were grown in DMEM supplemented with $10 \%$ FBS and 1\% Penicillin-Streptomycin Solution. In TOPFash luciferase reporter experiment, HEK293T cells were transfected $(24 \mathrm{~h}$ after $1 \times 10 \mathrm{e} 5$ seeding in 24-well plate, $80 \%$ confluence) with $375 \mathrm{ng}$ of WT or mutant WDR34/ empty vector plasmids, $125 \mathrm{ng}$ TOPFlashluciferase reporter, and $10 \mathrm{ng}$ of the Renilla-luciferase expression plasmid (Promega, Madison, WI, USA) per well using Lipofectamine 2000 (Thermo Fisher Scientific, Waltham, MA, USA). After $24 \mathrm{~h}$ in transfection media, cells were overlaid with WNT-3a conditioned media. Conditioned medium was harvested from WNT-3a secreting cells L Wnt-3A (CRL-2647, ATCC) per manufacturer's instructions. Cells were harvested after 12-h incubation and the Promega Dual Luciferase Assay System was used per manufacturer's instructions. pFR-luciferase reporter plasmid was used to evaluate PCP signaling. Cells were transfected with $65 \mathrm{ng}$ pFR-luciferase reporter, $60 \mathrm{ng}$ pFA2-cJun construct and $375 \mathrm{ng}$ various WDR34 constructs with $10 \mathrm{ng}$ Renilla-luciferase plasmid. Gli-luciferase reporter plasmid was used to evaluate SHH signaling. Cells were transfected with $65 \mathrm{ng}$ SHH plasmid or control pCMV6Entry plasmid, $200 \mathrm{ng}$ Gli reporter, and $240 \mathrm{ng}$ various
WDR34 constructs of either WT or mutant of p.G393S or p. Y437C with $10 \mathrm{ng}$ Renilla-luciferase plasmid. The firefly and Renilla-luciferase activities in cell lysates were measured $24 \mathrm{~h}$ after transfection for PCP and SHH signaling. At least three independent transfection experiments were performed, and each luciferase assay was performed in triplicate. Statistical significance was calculated using a twotailed $t$-test in Microsoft Excel.

\section{Western blot}

HEK293T cells were transfected with WT or mutant WDR34 in six-well plates using Lipofectamine 2000 (Thermo Fisher Scientific, USA) with a $0.5: 1$ ratio of reagent to DNA. After $36 \mathrm{~h}$, HEK293T cells were lysed in medium RIPA buffer (\#P0013C, Beyotime, China) with additional cocktail of protease inhibitors (Roche, Basel, Switzerland) and heated for $5 \mathrm{~min}$ at $100^{\circ} \mathrm{C}$. Cell lysates were analyzed by $10 \%$ SDS gel electrophoresis and transferred onto PVDF membranes (Merck Millipore, USA). Blocking of the membrane was done with 5\% nonfat milk in Tris-buffered saline containing $0.1 \%$ Tween-20. Then we incubated the membrane with mouse anti-Flag tag monoclonal antibody $(1: 1000 ; 66008-2-$ Ig; Proteintech, Chicago, IL, USA), mouse antibeta-actin monoclonal antibody (1:2000; A1978; Sigma-Aldrich, St. Louis, MO, USA) at $4{ }^{\circ} \mathrm{C}$ overnight, and then with Horseradish peroxidase-conjugated antimouse IgG secondary antibody (1:10,000; \#7076; Cell signaling technolog, USA) for 1 $\mathrm{h}$ at room temperature and visualized with a Tanon $138 \mathrm{Gel}$ imaging system (Tanon Science \& Technology, China). Band density was quantified with ImageJ and ratios of FlagWDR34 relative to $\beta$-actin were used to measure the expression of WT and mutant WDR34 protein. Three independent experiments were performed and representative results were shown.

\section{RT-qPCR}

HEK293T cells were transfected with WT or mutant WDR34 in six-well plates using Lipofectamine 2000 (Thermo Fisher Scientific, USA) with a 0.5:1 ratio of reagent to DNA. After $36 \mathrm{~h}$, total RNA from HEK293T cells were extracted by using Trizol reagent (\#15596026, Life technologies, USA).

Reverse transcription was performed with FastQuant RT Kit (With gDNase) (\#KR106-02, Tiangen Biotech (Beijing) Co., Ltd) and RT-qPCR was performed by using ChamQ Universal SYBR qPCR Master Mix kit (\#Q711-03, Vazyme, USA) to quantify the mRNA expression changes of SHH/PCP signaling target genes, including GLII and $J N K$. Expression levels were calculated using the $\Delta \Delta \mathrm{CT}$ method. GAPDH levels were used to normalize the genespecific expression levels. Student's $t$ test for paired samples 
was used to determine statistical significance. The sequence of primers are as follows:

GLII Forward: 5'-AGC GTG AGC CTG AAT CTG TG-3' GLII Reverse: 5'-CAG CAT GTA CTG GGC TTT GAA-3'

JNK Forward: 5'-TGT GTG GAA TCA AGC ACC TTC-3'

JNK Reverse: 5'-AGG CGT CAT CAT AAA ACT CGT TC-3'

WDR34 Forward: 5'-TCT GGG AAA GGT GAC GTG C-3'

WDR34 Reverse: 5'-CCC TTG TTC CGT GAA CTC TGT-3'

GAPDH Forward: 5'-CAT CAA GAA GGT GGT GAA GC- $3^{\prime}$

GAPDH Reverse: 5'-GTT GTC ATA CCA GGA AAT GAG C-3'

\section{Results}

\section{Identification of rare mutations of WDR34 in Chinese anencephaly cases}

We identified three heterozygous missense mutation in WDR34 c. $1066 \mathrm{G}>\mathrm{A}$ (p.G356S), c. $1177 \mathrm{G}>\mathrm{A}$ (p.G393S) and c. $1310 \mathrm{~A}>\mathrm{G}$ (p.Y437C) in four NTD patients by whole genome sequencing [26]. G393S were not previously reported in the 1000 Genome Project [27]. G393S and Y437C mutations were predicted as deleterious/damaging by SIFT and Polyphen-2 (Table 1). Therefore, we focused on these two potential disease causing mutations in the following study. Both G393 and Y437 are highly conserved amino acids located in WD domain (Fig. 1), suggesting that they are important for protein function.

\section{Both WDR34 mutations affect SHH signaling and G393S mutant also affect PCP signaling}

We first examined the effect of these mutants in protein expression level. Either mutations did not affect the expression level when they were overexpressed in HEK293T cells (Fig. 2a). As WDR34 is reported to be involved in SHH signaling, we tested the effect of these mutations on SHH signaling. Firstly, we performed luciferase reporter assay. As shown in Fig. 2b, overexpression of WT WDR34 significantly increased the SHH signaling when the Gli reporter is activated by SHH. The activated SHH signaling was significantly less than WT WDR34 when either G393S or Y437C was co-expressed. We also examined the expression level of endogenous GLII when HEK293T cells were transfected with either WT or mutant WDR34 expressing plasmids $(n=4)$. Similarly, the

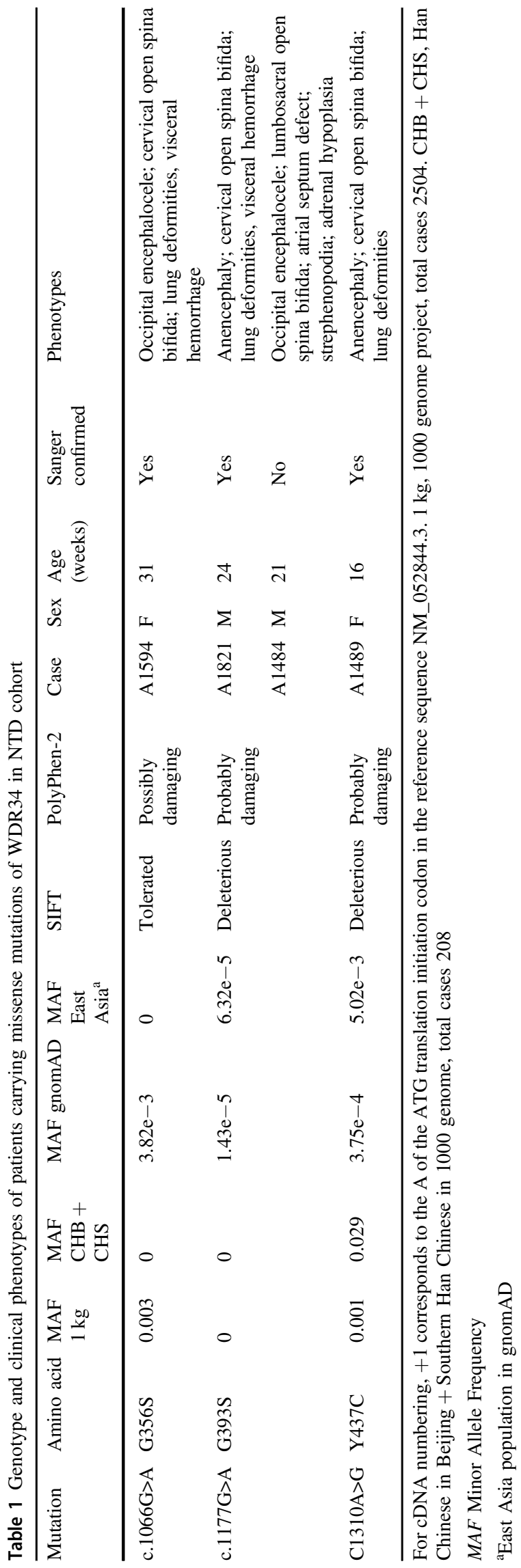


A

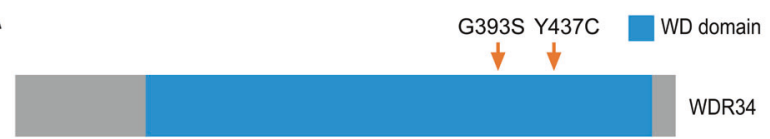

B

B c.1177G>A

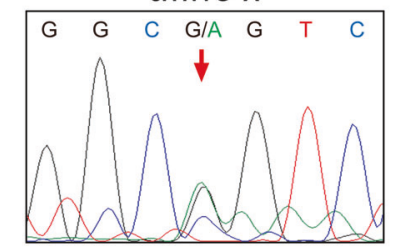

C

$\begin{array}{ll} & \text { c.1177G>A; p.G393S } \\ \text { Human } & \text { FTFSPHGGPI YSVSCSP } \\ \text { Chimpanzee } & \text { FTFSPHGGPI YSVSCSP } \\ \text { Horse } & \text { FTFSPHGGPVYSVSCSP } \\ \text { Cattle } & \text { FTFAPHGGPVYSVSCSP } \\ \text { Mouse } & \text { FTFSPHGGPVYSVSCSP } \\ \text { Norway rat } & \text { FTFSPHGGPVYSVSCSP } \\ \text { Chicken } & \text { LAFSPHS GPLYSVSCSP } \\ \text { Zebrafish } & \text { FSFSPRGGPI HSVHFSP }\end{array}$

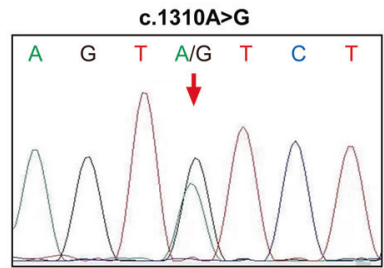

c.1310A>G; p.Y437C

LTSLQLSLK YLFAVRWSP LTSLQLSLK YLFAVRWSP LTSLQLSHKYLFAVRWSP LI SLQLSTK YLFCVRWSP LTSLQLSHKYLFAVRWSP LTSLQLSHKYLFAVRWSP LTSLQLSLK YLFAVRWSP LLALRVSDSYVFGVRWSP

Fig. 1 G393S and Y437C mutations in WDR34 were identified in our NTDs cohort. a Illustration of WDR34 protein structure, marked with two mutations identified in NTD cohorts. b Sequencing results of the WDR34 mutation. WD domain is colored blue. The arrows indicate the heterozygous nucleotides of each mutation. c A partial alignment of WDR34 amino acid sequence between human and several other vertebrates. The WDR34 variants found in NTDs affect highly conserved residues. NCBI accession numbers for WDR34 protein orthologs are: NP_443076.2 for human; XP_001160437.1 for chimpanzee; XP_023484820.1 for horse; NP_001193134.1 for cattle; NP_001008498.2 for mouse; NP_001005542.2 for Norway rat; XP_415494.2 for chicken; NP_001099160.1 for zebrafish

endogenous GLII expression level was significantly increased by WT WDR34 and such activation effect was lost when G393S or Y437C was overexpressed (Fig. 2c). Therefore, both G393S and Y437C mutants are loss-offunction mutations in SHH signaling. We also tested the effects of these two mutations on both canonical WNT and the planar cell polarity (PCP) signaling. The activity of WNT/ $\beta$-catenin signaling is detected by using the TOPFlash reporter, a $\beta$-catenin-responsive reporter. The PCP signaling is detected by the pFR and pFA2-c-Jun reporter system. Each reporter system was co-transfected with either WT or WDR34 mutants into HEK293T cells. The TOPFlash reporter activity was significantly inhibited by cotransfection of WT WDR34. When two mutants WDR34 were co-transfected, the inhibition effect on TOPFlash activity was not different from WT WDR34 (Fig. 2d). The PCP reporter luciferase activity was significantly increased by the co-transfection of WT and Y437C WDR34 $(n=3)$. But the PCP reporter activity is not changed with cotransfection of G393S WDR34 when compared to the control empty vector ( $n=4$, Fig. 2 e). To confirm the effect on PCP signaling, we also examined the expression level of endogenous $J N K$, a PCP downstream target gene, when

HEK293T cells were transfected with either WT or mutant WDR34 expressing plasmids $(n=4)$. As shown in Fig. 2f, the endogenous $J N K$ expression level was significantly elevated by WT WDR34 but such elevation was lost when G393S mutant was overexpressed. Our results showed that WT WDR34 could inhibit the WNT/ $\beta$-catenin signaling while activating PCP signaling in HEK293T cells (Fig. 2d-f). The Y437C mutant did not show any difference to WT WDR34 in either WNT/ $\beta$-catenin inhibition or PCP signaling activation. Surprisingly, the G393S mutant lost the ability to activate PCP signaling (Fig. 2e, f).

\section{Human WDR34 mutations Y437C and G393S caused neural tube and heart defects in zebrafish}

To investigate the in vivo effect of these WDR34 mutations on neural tube development, we used zebrafish model. MOs knockdown for zebrafish $w d r 34$ caused high ratio of NTDs (Fig. 3a, c) and pericardial abnormalities (Fig. 3b, e) in zebrafish embryos which are similar to previous report [32]. Within the MO injected fish, $63.4 \%$ showed abnormal curving and $60.5 \%$ showed dilated pericardium (Fig. 3c, e).

Either WT or mutated WDR34 were injected alone into zebrafish embryos to test the effect of overexpression of these proteins. A significantly higher frequency of NTDs and pericardial abnormalities were observed in zebrafish embryos injected with both WT and mutant WDR34 (Fig. 3d, f). We next conducted rescue experiment with either WT or mutant human WDR34 (Fig. 3c). We co-injected embryos with wdr34 MOs and either WT or mutant human WDR34 mRNA. The frequency of NTDs caused by MOs knockdown was significantly reduced by co-injection of WT WDR34. Both mutants had significantly less rescuing effects at the same concentration. To be noticed, at lower dosage $(115 \mathrm{ng})$, WT WDR34 did not increase the ratio of normal embryos, but it significantly reduced the ratio of embryos with severe phenotype and increased the ratio of embryos with mild phenotype. At this dosage, G393S did not have any rescuing effect; while Y437C had similar rescuing effect to WT WDR34 (Fig. 3c). For pericardial abnormalities, WT WDR34 significantly rescued the ratio of normal embryos at both dosages and Y437C had comparable rescuing ability to WT WDR34. Again, G393S mutation had much less rescuing effect on heart compared to WT WDR34 (Fig. 3e).

\section{Discussion}

In this study, we identified two potential disease causing mutations (c.1177G $>$ A; p.G393S and c.1310A $>$ G; p. Y437C) in WDR34 among a Chinese NTD cohort with mostly anencephaly cases. Surprisingly, WDR34 also 
A
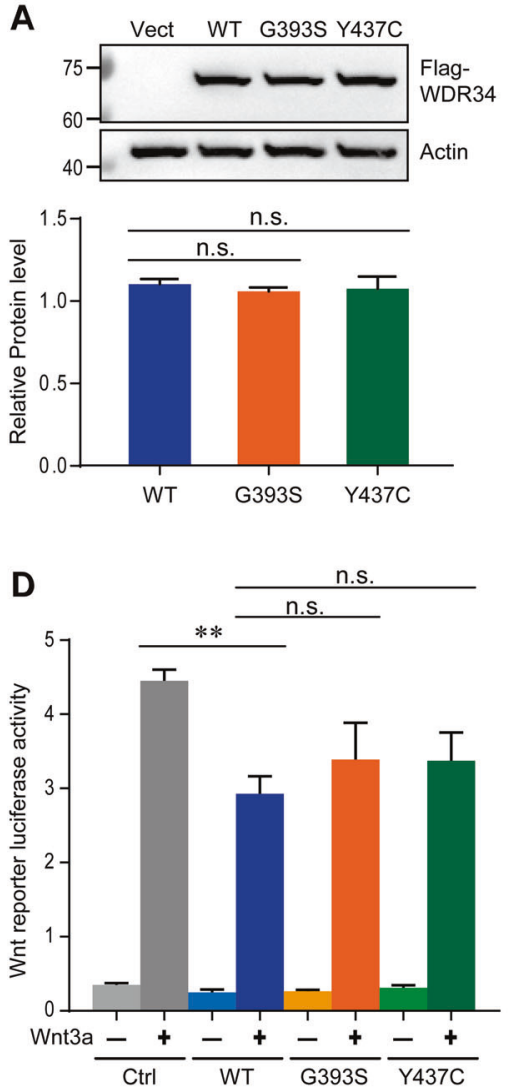

Fig. 2 Western blot analysis of WDR34 protein and signaling activity of WDR34. a HEK293T cells transfected with either WT or mutant WDR34 (Flag-tagged) plasmids. Cell lysates were blotted with antibody against either flag or actin, which serves as loading control. The representative WB images were shown in upper panel and the statistical analysis was shown in lower panel. $(n=3$, n.s. not significant $P \geq$ $0.05)$. The effects of G393S and Y437C in SHH (b, c), WNT/ $\beta$-catenin (d), and PCP signaling (e, f) by luciferase reporter assay or RT-qPCR. In luciferase assay, Gli reporter was used to indicate $\mathrm{SHH}$ signaling (b)
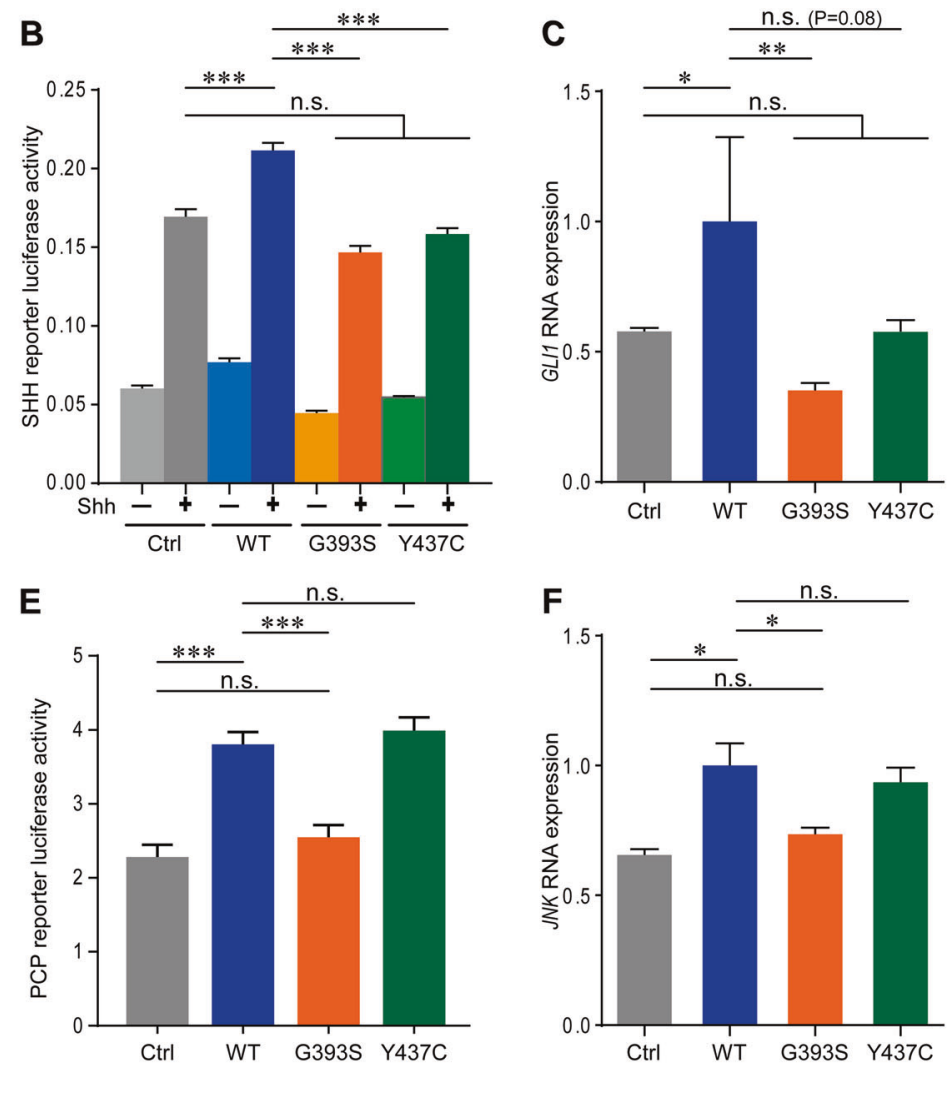

and TOPFlash Reporter was used to indicate WNT/ $\beta$-catenin signaling (d). The pFA2-c-Jun and JNK-responsive pFR-luciferase reporter was used to indicate PCP signaling (e). The effect on the endogenous GLII (c) and $J N K$ (f) expression levels were examined by RT-qPCR when HEK293T cells transfected with either WT or mutant WDR34 plasmids. The asterisk indicates a significant difference between WT and mutant WDR34. All experiments repeated for five times $(n=5)$ in luciferase assay and four time $(n=4)$ in RT-qPCR (n.s. not significant $P \geq 0.05 ; * P<0.05, * * P<0.01, * * * P<0.001$, two-tailed $t$-test) activates PCP signaling besides SHH signaling and the G393S is a loss-of-function mutation in both signaling pathways. G393S also showed less rescuing ability compared to WT and Y437C WDR34 in zebrafish. Our data suggested that WDR34 is involved in not only SHH but also PCP signaling, which is required for proper NTC.

As all known, SHH is important for neural tube patterning. Normally, the loss of activation of SHH signaling causes holoprosencephaly but not NTDs, However, the $W d r 34$ knockout mice exhibit exencephaly [25]. This is not fitting for the function of WDR34 in activating SHH signaling. On the other hand, mouse mutants with the loss of inhibition of SHH signaling causes exencephaly, such as Sufu knockout mouse [22]; and the Gli3 mutant Pdn/Pdn $[23,24]$. These data suggested that WDR34 is probably not only involved in SHH signaling pathway. NTC starts with convergent extension (CE), which is the process of lengthening and narrowing of the initially disc-shaped neural plate [33]. CE process depends on a noncanonical WNT signaling cascade, the PCP pathway [34]. Mutations in PCP genes, such as VANGL1 [35] and VANGL2 [36], have been reported to be associated with NTDs in human. Mouse models with mutations in the genes of the PCP pathway also showed very severe NTDs, including craniorachischisis and exencephaly [37, 38]. Here we also observed that overexpression of WDR34 could activate PCP signaling and G393S mutant is a loss-of-function mutant in PCP signaling activation. Therefore, the exencephaly phenotypes in $W d r 34^{-l-}$ mice is likely more due to its function in the PCP pathway. What is more, although zebrafish does not have similar neurulation process to human or mouse $[39,40]$, it is an excellent model to study CE and the conserved PCP signaling during its neural tube development [41]. The MO knockdown of $w d r 34$ in zebrafish showed CE defects in neural tube (Fig. 3), which is also collaborates with the function of WDR34 in PCP signaling. 
Fig. $3 \mathrm{MO}$ of $w d r 34$ induced NTD and pericardial abnormalities in zebrafish embryos. a The severity of the NTD was determined based on the morphogenesis of the neural tube as either curved NTDs or shorten spine. b The enlarged pericardial is indicated by red asterisk. Scale bar in (a) \& (b) are $200 \mu \mathrm{m}$. Injection of $w d r 34$ MOs yielded high proportion NTD (c) and pericardial abnormalities (e) phenotypes in zebrafish. G393S exhibited lower rescuing ability than WT mutations. Overexpression of WT and mutant WDR34 alone also induced NTDs (d) and pericardial abnormalities (f) in zebrafish. The number above each bar is the total number of embryos examined under each experimental condition. The $P$ value was calculated by twotailed Fisher's exact test. The asterisk indicates a statistically significant difference (n.s. not significant $P \geq 0.05 ; * P<0.05$, $* * P<0.01, * * * P<0.001)$
A

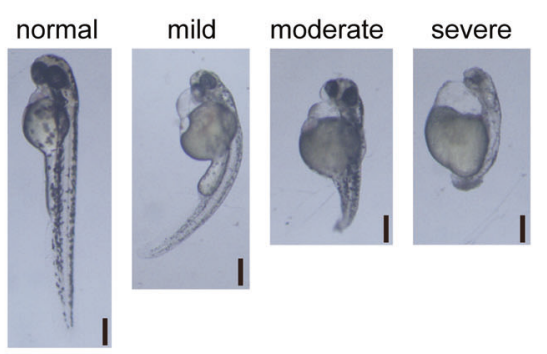

C

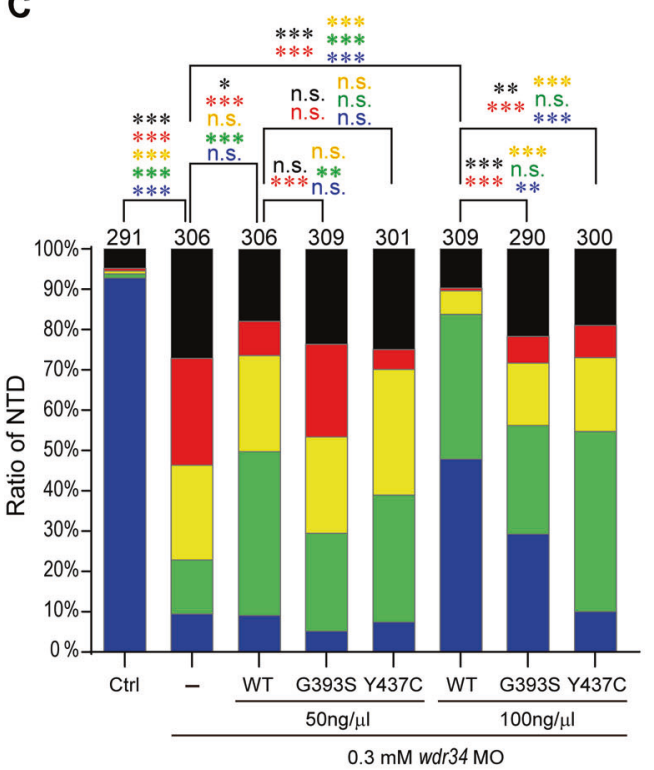

E

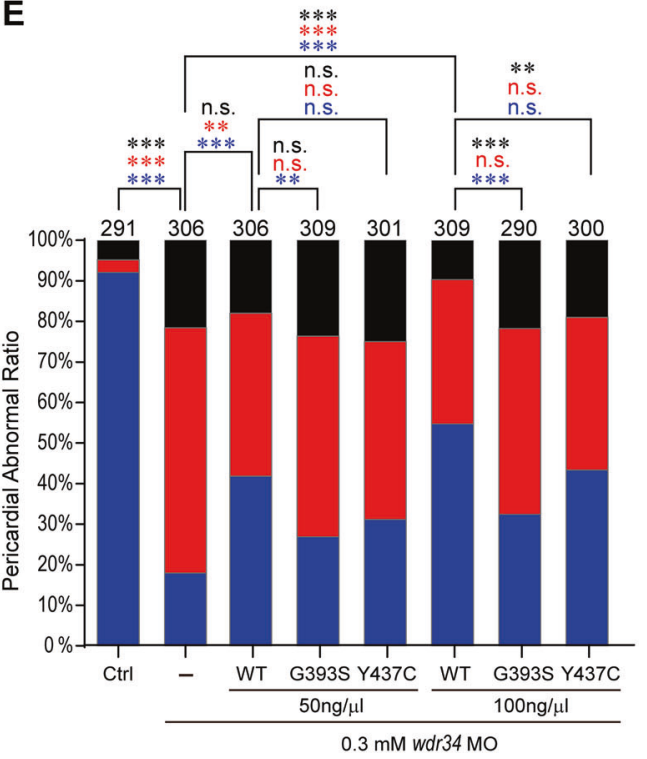

B

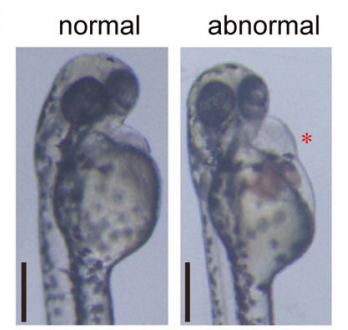

D

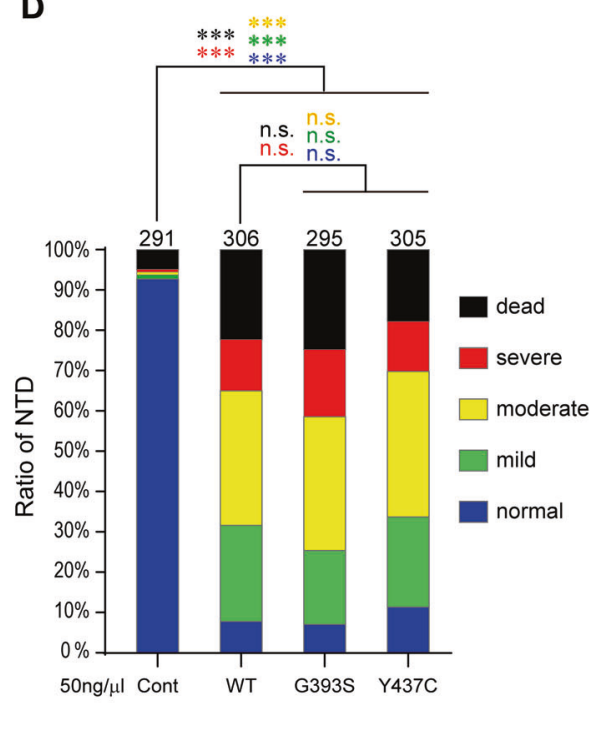

$\mathbf{F}$

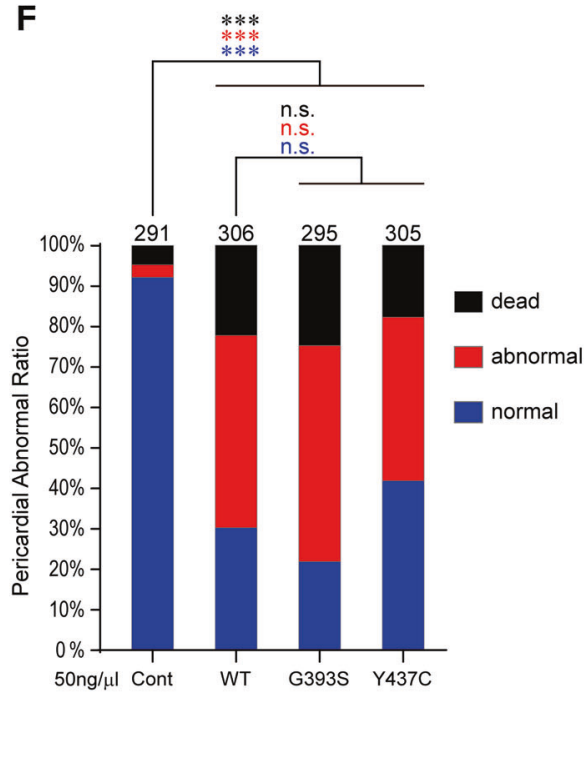

Homozygosity and compound heterozygosity of missense mutations in the WDR34 gene have been associated with SRTD11 with or without polydactyly (OMIM number *613363) [16, 17]. The G393S mutant was previously identified in two Turkish asphyxiating thoracic dystrophy patients [17]. One patient has homozygosity of G393S and the other patient has compound heterozygosity of G393S with a T514Rfs*11 mutation. Our study is the first report of WDR34 mutations associated with NTDs in human. Based on the structure of WDR34 [17, 42], the $\beta$-propeller domain of WDR34 is crucial for its interaction with other dynein-2 components such as the dynein heavy chain. G393 is located on the surface of the $\beta$-propeller domain, which may interfere its interaction with other dynein-2 components. 
Although Y437 is also located in the $\beta$-propeller domain, it is not as close to the surface and on different side of the $\beta$ propeller, which might explain its functional difference to G393 and higher frequency in general populations (Table 1).

We also observed a slight inhibition of canonical WNT signaling by WDR34 in HEK293T cells (Fig. 2c). A recent report showed that WDR34 activates canonical WNT signaling in hepatocellular carcinoma [43]. The reason for the different effects of WDR34 on canonical WNT signaling between our HEK293T cells and their hepatocellular carcinoma cells may due to differences in cell systems, changes between development and cancer, etc. Interestingly, they showed interaction of WDR34 to the WNT receptor Frizzled (Fz). WDR34 also enhances the interaction between Fz and Dvl2 [43]. To be notice, Dvl2 is also a key component of PCP signaling [44] and mutations in DVL2 are associated with NTDs in human [45]. Further investigation will be needed to understand how WDR34 regulate different signaling pathways.

We observed pericardial abnormalities in our zebrafish experiments. Despite the difference between zebrafish and human, the pericardial abnormalities still suggested that WDR34 may be also involved in heart development and associated with congenital heart disease (CHD). A recent forward genetic screening on mutagenesis mouse library also suggested that "cilia and cilia-transduced cell signaling have important roles in the pathogenesis of CHD" [46]. Although currently there is no report on WDR34 to be associated with CHD, it would be interesting to dig deeper in this matter.

In conclusion, here we reported rare mutations of WDR34 identified in Chinese anencephaly patients. Our results showed a new function of WDR34 in activating PCP signaling. Mutation in WDR34 could contribute to human NTDs by affecting not only SHH but also PCP signaling.

Acknowledgements This work was supported by grants to YZ from the National Natural Science Foundation of China (81870894, 81741048), the National Key Research and Development Program (2018YFA0800303); and Innovation Program of Shanghai Municipal Education Commission (2019-01-07-00-07-E00041).

Author contributions $\mathrm{YZ}$ designed the study and wrote the paper. HY performed the Sanger sequencing, luciferase, and zebrafish experiments, RP and ZC performed sequence analysis. TZ and HW recruited the patients. All authors reviewed the paper and contributed to scientific content.

\section{Compliance with ethical standards}

Conflict of interest The authors declare that they have no conflict of interest.

Publisher's note Springer Nature remains neutral with regard to jurisdictional claims in published maps and institutional affiliations.

\section{References}

1. Blencowe H, Kancherla V, Moorthie S, Darlison MW, Modell B. Estimates of global and regional prevalence of neural tube defects for 2015: a systematic analysis. Ann N Y Acad Sci. 2018;1414:31-46.

2. Mitchell LE. Epidemiology of neural tube defects. Am J Med Genet C Semin Med Genet. 2005;135C:88-94.

3. Botto LD, Moore CA, Khoury MJ, Erickson JD. Neural-tube defects. N Engl J Med. 1999;341:1509-19.

4. Li Z, Ren A, Zhang L, Ye R, Li S, Zheng J, et al. Extremely high prevalence of neural tube defects in a 4-county area in Shanxi Province, China. Birth Defects Res Part A Clin Mol Teratol. 2006;76:237-40.

5. Li Y, Lu W, He X, Bu G. Modulation of LRP6-mediated Wnt signaling by molecular chaperone Mesd. FEBS Lett. 2006;580:5423-8.

6. Wallingford JB, Niswander LA, Shaw GM, Finnell RH. The continuing challenge of understanding, preventing, and treating neural tube defects. Science.2013;339:1222002.

7. Leck I. Causation of neural tube defects: clues from epidemiology. Br Med Bull. 1974;30:158-63.

8. Goetz SC, Anderson KV. The primary cilium: a signalling centre during vertebrate development. Nat Rev Genet. 2010;11:331-44.

9. Anvarian Z, Mykytyn K, Mukhopadhyay S, Pedersen LB, Christensen ST. Cellular signalling by primary cilia in development, organ function and disease. Nat Rev Nephrol. 2019;15:199-219.

10. He M, Agbu S, Anderson KV. Microtubule motors drive hedgehog signaling in primary cilia. Trends Cell Biol. 2017;27:110-25.

11. Roberts AJ. Emerging mechanisms of dynein transport in the cytoplasm versus the cilium. Biochem Soc Trans. 2018;46:967-82.

12. Hildebrandt F, Benzing T, Katsanis N. Ciliopathies. N Engl J Med. 2011;364:1533-43.

13. Reiter JF, Leroux MR. Genes and molecular pathways underpinning ciliopathies. Nat Rev Mol Cell Biol. 2017;18:533-47.

14. Asante D, Stevenson NL, Stephens DJ. Subunit composition of the human cytoplasmic dynein-2 complex. J Cell Sci. 2014;127:4774-87.

15. Tsurumi Y, Hamada Y, Katoh Y, Nakayama K. Interactions of the dynein-2 intermediate chain WDR34 with the light chains are required for ciliary retrograde protein trafficking. Mol Biol Cell. 2019;30:658-70.

16. Huber C, Wu S, Kim AS, Sigaudy S, Sarukhanov A, Serre V, et al. WDR34 mutations that cause short-rib polydactyly syndrome type III/severe asphyxiating thoracic dysplasia reveal a role for the NF-kappaB pathway in cilia. Am $\mathrm{J}$ Hum Genet. 2013;93:926-31.

17. Schmidts M, Vodopiutz J, Christou-Savina S, Cortes CR, McInerney-Leo AM, Emes RD, et al. Mutations in the gene encoding IFT dynein complex component WDR34 cause Jeune asphyxiating thoracic dystrophy. Am J Hum Genet. 2013;93:932-44.

18. Ruiz i Altaba A, Nguyen V, Palma V. The emergent design of the neural tube: prepattern, SHH morphogen and GLI code. Curr Opin Genet Dev. 2003;13:513-21.

19. Patten I, Placzek M. The role of Sonic hedgehog in neural tube patterning. Cell Mol Life Sci. 2000;57:1695-708.

20. Wilson L, Maden M. The mechanisms of dorsoventral patterning in the vertebrate neural tube. Dev Biol. 2005;282:1-13.

21. Murdoch JN, Copp AJ. The relationship between sonic Hedgehog signaling, cilia, and neural tube defects. Birth Defects Res Part A Clin Mol Teratol. 2010;88:633-52.

22. Svard J, Heby-Henricson K, Persson-Lek M, Rozell B, Lauth M, Bergstrom A, et al. Genetic elimination of Suppressor of fused 
reveals an essential repressor function in the mammalian Hedgehog signaling pathway. Dev Cell. 2006;10:187-97.

23. Maekawa M, Ohta K, Katagiri R, Ueta E, Naruse I. Exencephaly induction by valproic acid in the genetic polydactyly/arhinencephaly mouse, Pdn/Pdn. Congenit Anom. 2005;45:132-6.

24. Naruse I, Ueta E. Hydrocephalus manifestation in the genetic polydactyly/arhinencephaly mouse ( $\mathrm{Pdn} / \mathrm{Pdn})$. Congenit Anom. 2002;42:27-31.

25. Wu C, Li J, Peterson A, Tao K, Wang B. Loss of dynein-2 intermediate chain Wdr34 results in defects in retrograde ciliary protein trafficking and Hedgehog signaling in the mouse. Hum Mol Genet. 2017;26:2386-97.

26. Chen Z, Lei Y, Zheng Y, Aguiar-Pulido V, Ross ME, Peng R, et al. Threshold for neural tube defect risk by accumulated singleton loss-of-function variants. Cell Res. 2018;28:1039-41.

27. Genomes Project C, Auton A, Brooks LD, Durbin RM, Garrison EP, Kang HM, et al. A global reference for human genetic variation. Nature. 2015;526:68-74.

28. Lek M, Karczewski KJ, Minikel EV, Samocha KE, Banks E, Fennell T, et al. Analysis of protein-coding genetic variation in 60,706 humans. Nature. 2016;536:285-91.

29. Adzhubei IA, Schmidt S, Peshkin L, Ramensky VE, Gerasimova A, Bork P, et al. A method and server for predicting damaging missense mutations. Nat Methods. 2010;7:248-9.

30. Sim NL, Kumar P, Hu J, Henikoff S, Schneider G, Ng PC. SIFT web server: predicting effects of amino acid substitutions on proteins. Nucleic Acids Res. 2012;40:W452-7.

31. Kumar P, Henikoff S, Ng PC. Predicting the effects of coding non-synonymous variants on protein function using the SIFT algorithm. Nat Protoc. 2009;4:1073-81.

32. Krock BL, Mills-Henry I, Perkins BD. Retrograde intraflagellar transport by cytoplasmic dynein-2 is required for outer segment extension in vertebrate photoreceptors but not arrestin translocation. Investig Ophthalmol Vis Sci. 2009;50:5463-71.

33. Ybot-Gonzalez P, Savery D, Gerrelli D, Signore M, Mitchell CE, Faux $\mathrm{CH}$, et al. Convergent extension, planar-cell-polarity signalling and initiation of mouse neural tube closure. Development. 2007;134:789-99.

34. Copp AJ, Greene ND. Neural tube defects-disorders of neurulation and related embryonic processes. Wiley Interdiscip Rev Dev Biol. 2013;2:213-27.
35. Kibar Z, Torban E, McDearmid JR, Reynolds A, Berghout J, Mathieu M, et al. Mutations in VANGL1 associated with neuraltube defects. N Engl J Med. 2007;356:1432-7.

36. Lei YP, Zhang T, Li H, Wu BL, Jin L, Wang HY. VANGL2 mutations in human cranial neural-tube defects. N Engl J Med. 2010;362:2232-5.

37. Juriloff DM, Harris MJ. A consideration of the evidence that genetic defects in planar cell polarity contribute to the etiology of human neural tube defects. Birth Defects Res Part A Clin Mol Teratol. 2012;94:824-40.

38. Copp AJ, Stanier P, Greene ND. Neural tube defects: recent advances, unsolved questions, and controversies. Lancet Neurol. 2013;12:799-810.

39. Araya C, Ward LC, Girdler GC, Miranda M. Coordinating cell and tissue behavior during zebrafish neural tube morphogenesis. Dev Dyn. 2016;245:197-208.

40. Cearns MD, Escuin S, Alexandre P, Greene ND, Copp AJ. Microtubules, polarity and vertebrate neural tube morphogenesis. J Anat. 2016;229:63-74.

41. Jussila M, Ciruna B. Zebrafish models of non-canonical Wnt/planar cell polarity signalling: fishing for valuable insight into vertebrate polarized cell behavior. Wiley Interdiscip Rev Dev Biol. 2017;6:3.

42. Toropova K, Zalyte R, Mukhopadhyay AG, Mladenov M, Carter AP, Roberts AJ. Structure of the dynein-2 complex and its assembly with intraflagellar transport trains. Nat Struct Mol Biol. 2019;26:823-9.

43. Luo X, Liu Y, Ma S, Liu L, Xie R, Wang S. WDR34 activates Wnt/Beta-catenin signaling in hepatocellular carcinoma. Dig Dis Sci. 2019;64:2591-9.

44. Yan D, Wallingford JB, Sun TQ, Nelson AM, Sakanaka C, Reinhard $\mathrm{C}$, et al. Cell autonomous regulation of multiple Dishevelled-dependent pathways by mammalian Nkd. Proc Natl Acad Sci USA. 2001;98:3802-7.

45. De Marco P, Merello E, Consales A, Piatelli G, Cama A, Kibar Z, et al. Genetic analysis of disheveled 2 and disheveled 3 in human neural tube defects. J Mol Neurosci. 2013;49:582-8.

46. Li Y, Klena NT, Gabriel GC, Liu X, Kim AJ, Lemke K, et al. Global genetic analysis in mice unveils central role for cilia in congenital heart disease. Nature. 2015;521:520-4. 